\title{
Les difficultés de régénération naturelle des pessières subalpines: prédation des graines au sol et blocages dus à l'humus
}

\author{
F Pellissier, L Trosset
}

\begin{abstract}
Université de Savoie, Institut savoisien de la montagne, département de biologie, dynamique des écosystèmes d'altitude, BP 1104, 73011 Chambéry Cedex, France
\end{abstract}

(Reçu le 15 juillet 1991; accepté le 14 janvier 1992)

\begin{abstract}
Résumé - Les peuplements d'épicéa de l'étage subalpin sont caractérisés par une régénération naturelle déficiente. Afin de cerner les obstacles qui s'opposent à l'installation et au développement des jeunes plantules, des semis expérimentaux ont été réalisés en différentes pessières des Alpes nord-occidentales. Les différentes modalités appliquées lors de cette expérimentation ont mis en évidence un impact important de la prédation des graines au sol par divers rongeurs et des blocages de la germination dus à l'humus. L'influence de ce dernier facteur persiste les années suivant la levée des semis et se concrétise par une disparition considérable des jeunes plants.
\end{abstract}

Picea abies L Karst / régénération / Alpes / forêts d'altitude / semis / prédation / humus

Summary - Difficulty of natural regeneration of subalpine forests: seed consumption and humic inhibition. The subalpine spruce forest is characterized by deficient natural regeneration. To determine which factors were detrimental to the installation and growth of young saplings, experimental seedlings were sown at different sites (Adenostylo alliariae-Piceetum and HomogynoPiceetum) in the northwestern Alps (table 1). Combinations of several types of treatment in the seed beds allowed determination of the optimal conditions for installation and establishment of the most favourable ecological conditions for seedling survival. Predation by rodents and humic parameters (aluminium level) were found to restrict seed germination (table II). The latter factor was also responsible for an important loss of plantlets (at least 50\%) after the first year (table II).

Picea abies L / regeneration / Alps / altitudinal forest / seedling / predation / humus 


\section{INTRODUCTION}

Les forêts des Alpes nord-occidentales sont composées, pour une grande part, de conifères au sein desquels l'épicéa (Picea abies $L$ Karst) occupe une place prépondérante $(63 \%$ de la surface enrésinée en Savoie). Cette essence forme, dans les étages montagnard supérieur et subalpin, de grands peuplements pratiquement monospécifiques, caractérisés par une régénération naturelle déficiente (André et $a l$, 1986). Ce constat s'applique non seulement aux départements voisins (HauteSavoie et Isère) mais aussi à l'ensemble des pays de l'arc alpin : Suisse (Sorg, 1980), Allemagne (Mayer, 1986) et Italie (Piussi, 1986). Cette difficulté de rajeunissement remet en cause la pérennité de la couverture végétale qui assure la stabilité des versants (protection contre l'érosion et les avalanches). Face à la réussite aléatoire et au coût des reboisements d'altitude, il apparaît souhaitable de reconsidérer l'intérêt de la régénération naturelle, tant sur le plan fondamental (connaissance des mécanismes responsables du dysfonctionnement), qu'appliqué (sylviculture adaptée au contexte montagnard).

II s'avère donc nécessaire de préciser les conditions d'installation du jeune plant. Pour cela, des semis expérimentaux ont été réalisés en 1986 et suivis jusqu'en 1989 : les résultats font l'objet de cet article. La problématique de cette étude prend en considération la prédation des graines au sol (Vincent, 1977), le rôle potentiel de l'humus dans les phénomènes de blocage (Pellissier et Trosset, 1989a) et la concurrence nutritionnelle et énergétique de la végétation en sous-strate (Gregori, 1967; Roussel, 1969). L'intérêt porté à ces trois facteurs nous a amenés à choisir nos sites d'étude dans deux types de peuplements bien démarqués : la pessière à myrtille (Homogyno-Piceetum selon Mayer 1986) et la pessière à hautes herbes (Adenostylo alliariae-Piceetum, Mayer 1986). Les sites expérimentaux sont localisés dans les forêts communales de Mâcot (parcelles 5 et 19), Cohennoz (parcelles $D$ et $K$ ), Notre-Dame-deBellecombe (parcelles $A$ et $B$ ) et EssertsBlay (parcelle 24), en Savoie.

Ces premiers travaux permettent de dégager les principaux facteurs s'opposant à une installation optimale de semis naturels et de proposer quelques recommandations aux praticiens, en vue de favoriser la régénération naturelle.

\section{MATÉRIEL ET MÉTHODES}

Pour chaque parcelle, on sélectionne une aire représentative du peuplement (d'environ 1 ha) et homogène quant aux caractéristiques phytosociologiques et pédologiques (tableau I). On y réalise 5 placettes expérimentales, de $5 \mathrm{~m}^{2}$ chacune $(5 \times 1 \mathrm{~m})$, selon les modalités définies cidessous :

- A : témoin. Pas de perturbation du sol, ni de la végétation;

- B : élimination de la végétation concurrente. Coupe au ras du sol des strates herbacée et sous-arbustive;

- C : B + crochetage de l'humus;

- D : B + protection par grillage (maille de 5 $\mathrm{mm}$ );

- E : C + protection par grillage (maille de 5 $\mathrm{mm})$.

On définit par "crochetage" la scarification à la pioche de l'horizon humifère.

Entre le $1^{\text {er }}$ et le 20 juin 1986, c'est-à-dire sitôt la neige fondue, 200 graines $/ \mathrm{m}^{2}$ (fournies par les sécheries de la Joux, provenance Hautes Chaînes du Jura) ont été semées. Leur pouvoir germinatif, déterminé au laboratoire, est de $75 \%$. Les résultats présentés intègrent la correction due au pouvoir germinatif (report à $100 \%$ ). Quatre mois plus tard, on compte le nombre de plantules. Les années suivantes (1987, 1988 et 1989), on dénombre les plantules survivantes. L'absence de grainée durant ces 3 années a facilité l'expérimentation, aucun semis naturel ne venant perturber les comptages. 
Tableau I. Principales caractéristiques phytosociologiques et pédologiques des sites expérimentaux. Le chiffre (ou la lettre) indiqué entre parenthèses à la suite du nom de la commune répertorie la parcelle forestière selon le cahier d'aménagement de l'ONF.

\begin{tabular}{|c|c|c|c|c|c|}
\hline Site & Pessière & Humus & $(\mathrm{S} / \mathrm{T}) \times 100$ & $\begin{array}{c}A l^{3+} \\
\text { (meq/100 g } \\
\text { sol) }\end{array}$ & $\begin{array}{c}\mathrm{H}^{+} \\
(\mathrm{meq} / 100 \mathrm{~g} \\
\text { sol) }\end{array}$ \\
\hline Cohennoz (D) & Adenostylo alliariae-Piceetum & Mull & 73,8 & 3,2 & 1,6 \\
\hline ND Bellecombe (B) & Adenostylo alliariae-Piceetum & Mull & 85,4 & 2,7 & 0,5 \\
\hline Cohennoz (K) & Homogyno-Piceetum & Mor & 21,3 & 21,1 & 1,8 \\
\hline Esserts-Blay (24) & Homogyno-Piceetum & Mor & 28,6 & 20,5 & 3,1 \\
\hline Mâcot (5) & Homogyno-Piceetum & Mor & 30,1 & 13,5 & 2,4 \\
\hline Mâcot (19) & Homogyno-Piceetum & Mor & 45,4 & 11,1 & 2,8 \\
\hline ND Bellecombe (A) & Homogyno-Piceetum & Mor & 17,8 & 23,6 & 2,1 \\
\hline
\end{tabular}

Les résultats sont exprimés par le taux de germination (G), la première année et le taux de survie des plantules (S), les années suivantes :

$$
G=\frac{\text { Nombre de plantules }}{\text { Nombre de graines semées }} \text { pouvoir germinatif }
$$

Nombre de plantules en 1987,1988 ou 1989 $S$

Nombre de plantules en 1986

Les résultats sont traités par deux analyses de variance, une première pour le taux de germination et une seconde pour la survie. Dans les deux cas, les traitements appliqués constituent le facteur étudié et le site expérimental, le facteur bloc. Pour le taux de germination, la variable analysée est le nombre de plantules comptabilisé au premier automne (1986), tandis que pour le taux de survie, on considère trois variables : les taux de survie en 1987, 1988 et 1989. Dans tous les cas, un test de NewmanKeuls (au seuil de $5 \%$ ), réalisé à la suite de l'analyse de variance, permet de classer les traitements par groupes homogènes.

\section{RÉSULTATS}

Le tableau II regroupe les taux de germination et de survie, en fonction des traitements appliqués, pour chacun des sites.
II n'existe pas d'effet-bloc : bien que les sites expérimentaux aient été choisis dans deux secteurs géographiques distincts par leurs particularités climatiques (Préalpes et Alpes internes), celles-ci n'interviennent pas dans l'explication des différences observées.

\section{Taux de germination}

Les témoins présentent un taux de germination proche de zéro, confirmant ainsi les difficultés de régénération naturelle. La suppression de la végétation (traitement $B$ ) n'augmente pas de manière significative le nombre de plantules présentes : le phénomène de concurrence ne semble pas intervenir de manière importante durant les premiers mois qui suivent la germination. Le test de Newman-Keuls regroupe les traitements $A$ et $B$ : une simple élimination de la végétation ne suffit donc pas à favoriser l'installation des semis. Le même test individualise, par contre, les traitements $C$, $D$ et $E$ des modalités $A$ et $B$ : le remaniement de l'humus (C) et la protection des graines vis-à-vis des rongeurs (D) apportent une réponse positive au problème de la levée des semis. Les meilleurs résultats 
Tableau II. Taux de germination (1986) et de survie des plants (1987 à 1989) de chacun des sites expérimentaux, pour les différents traitements appliqués. (1) : Le taux de survie est indéterminé, en raison du taux de germination nul.

Traitements

\begin{tabular}{|c|c|c|c|c|c|c|}
\hline Site & Année & $A$ & $B$ & $c$ & $D$ & $E$ \\
\hline \multicolumn{7}{|c|}{ Cohennoz (D) } \\
\hline & 1986 & $0,004 \pm 0,004$ & $0,003 \pm 0,003$ & $0,071 \pm 0,02$ & $0,285 \pm 0,03$ & $0,337 \pm 0,09$ \\
\hline & 1987 & $0,750 \pm 0,167$ & $0,030 \pm 0,07$ & $0,730 \pm 0,16$ & $0,460 \pm 0,04$ & $0,280 \pm 0,18$ \\
\hline & 1988 & 0 & 0 & $0,360 \pm 0,17$ & $0,160 \pm 0,04$ & $0,100 \pm 0,19$ \\
\hline & 1989 & 0 & 0 & 0 & $0,020 \pm 0,02$ & $0,010 \pm 0,03$ \\
\hline
\end{tabular}

Cohennoz (K)

1986

1987

1988

$\begin{array}{ll}0,07 \pm 0,08 & 0,020 \pm 0,006 \\ 0,360 \pm 0,06 & 0 \\ 0,090 \pm 0,06 & 0 \\ 0 & 0\end{array}$

$0,160 \pm 0,002$

$0,130 \pm 0,02$

$0,260 \pm 0,03$

$0,180 \pm 0,01$

$0,040 \pm 0,02$

$0,410 \pm 0,2$

$0,850 \pm 0,1$

1989

0

$0,130 \pm 0,09$

$0,020 \pm 0,01$

$0,200 \pm 0,09$

$0,160 \pm 0,08$

Esserts-Blay (24)

1986

1987

0

$-(1)$

0

1988

$-(1)$

$0,040 \pm 0,009$

$0,900 \pm 0,1$

$0,470 \pm 0,32$

$0,160 \pm 0,03$

$0,640 \pm 0,16$

1989

$-$

- $\quad 0,470 \pm 0,32$

$0,570 \pm 0,17$

$0,290 \pm 0,09$

$0,210 \pm 0,025$

$0,680 \pm 0,12$

$0,230 \pm 0,07$

$0,220 \pm 0,07$

Mâcot (5)

1986

1987

$0,003 \pm 0,002 \quad 0,009 \pm 0,005$ $0,440 \pm 0,09$

$0,520 \pm 0,36$

$0,040 \pm 0,02$

$0,940 \pm 0,12$

$0,240 \pm 0,1$

$0,280 \pm 0,17$

$0,410 \pm 0,12$

$0,200 \pm 0,13$

$0,009 \pm 0,006$

$0,270 \pm 0,06$

$0,940 \pm 0,21$

$0,710 \pm 0,07$

0

0

$0,400 \pm 0,07$

$0,190 \pm 0,09$

Mâcot (19)

$\begin{array}{ll}1986 & 0,060 \pm 0,03 \\ 1987 & 0,010 \pm 0,01 \\ 1988 & 0 \\ 1989 & 0\end{array}$

$0,070 \pm 0,03$
$0,300 \pm 0,23$
$0,130 \pm 0,14$
$0,030 \pm 0,04$

$0,100 \pm 0,02$

$0,500 \pm 0,14$

$0,040 \pm 0,02$

$0,020 \pm 0,02$

$0,080 \pm 0,03$

0

$0,210 \pm 0,07$

$0,040 \pm 0,03$

0

$0,340 \pm 0,09$

$0,240 \pm 0,09$

$0,090 \pm 0,1$

ND Bellecombe (A)

$\begin{array}{llll}1986 & 0 & 0,004 \pm 0,001 & 0,090 \pm 0,006 \\ 1987 & -(1) & 0 & 0,450 \pm 0,05 \\ 1988 & - & 0 & 0,410 \pm 0,05 \\ 1989 & - & 0 & 0,380 \pm 0,07\end{array}$

$0,130 \pm 0,004$

$0,270 \pm 0,009$

$0,780 \pm 0,09$

$0,820 \pm 0,11$

$0,360 \pm 0,11$

$0,710 \pm 0,13$

$0,350 \pm 0,1$

$0,580 \pm 0,09$

ND Bellecombe (B)

$\begin{array}{llllll}1986 & 0,007 \pm 0,002 & 0,001 \pm 0,001 & 0,053 \pm 0,02 & 0,173 \pm 0,04 & 0,284 \pm 0,011 \\ 1987 & 0 & 0,430 \pm 0,09 & 0,740 \pm 0,04 & 0,330 \pm 0,04 & 0,530 \pm 0,018 \\ 1988 & 0 & 0 & 0,660 \pm 0,05 & 0,220 \pm 0,06 & 0,490 \pm 0,02 \\ 1989 & 0 & 0 & 0,580 \pm 0,07 & 0,030 \pm 0,009 & 0,110 \pm 0,04\end{array}$


sont obtenus en couplant ces deux traitements (E).

\section{Taux de survie}

Pour les 3 années de mesure, le traitement statistique fait apparaître des comportements similaires pour les modalités $A$ et B d'une part, et C, D et E d'autre part (le faible taux de survie observé pour la modalité $E$ de la parcelle $D$ de Cohennoz s'explique par une fonte de semis d'origine parasitaire). II faut cependant se garder de tirer des conclusions trop hâtives : le taux de survie nul des modalités $A$ et $B$ résulte simplement d'une levée des semis très faible en 1986 !

Mises à part les stations d'Esserts-Blay, Notre-Dame de Bellecombe (A) et Mâcot (5), le taux de survie au terme de la $3^{e}$ année est très faible, quel que soit le traitement appliqué : la préparation du sol et la protection grillagée deviennent donc inefficaces et la survie des jeunes plants dépend certainement plus des caractéristiques écologiques de la parcelle.

\section{DISCUSSION}

Les comptages effectués à la fin de la première année dans chacun des sites révèlent une prédation importante des graines au sol et un blocage de la germination imputable à l'humus.

Concernant la prédation, peu d'études ont été menées sur les essences résineuses : Smith et Balda, 1979; Genard et Lescourret, 1984 démontrent le rôle de divers micromammifères en ce domaine. L'observation de nombreuses graines vidées de leur contenu et dont les téguments étaient percés sur un côté, laissent supposer que les responsables de tels dégâts sont des Muridae : Apodemus sylvati- cus, A flavicollis... particulièrement nombreux dans ces peuplements (Bergeret, 1986).

À cela s'ajoute le rôle d'autres prédateurs potentiels (écureuils, oiseaux...), non quantifié dans cette étude. L'impact de ces divers agents sur le stock de graines viables parvenant au sol est donc loin d'être négligeable et une méthode de lutte efficace doit être recherchée.

L'influence de l'humus intervient à la fois sur la germination et la survie des plants. L'expérience présentée ici ne permet pas de préciser les mécanismes d'un tel blocage mais des travaux antérieurs (Pellissier et Trosset, 1989b) mettent en évidence une inhibition allélopathique de la germination de l'épicéa dans les humus de type mor Drapier (1983), démontre in situ l'auto-intoxication du sapin (pluviolessivats), dans les sapinières vosgiennes et propose donc de faciliter la régénération naturelle par alternance des essences. Par ailleurs, certains auteurs (Van Praag et al, 1985a, b; Bonneau et al, 1987) soulignent la toxicité aluminique sur le développement racinaire et la difficulté à réaliser rapidement la mycorhization (Rost-Siebert, 1985). Dans le cas présent, on peut envisager semblables phénomènes, dès la seconde année. En effet, la détermination des cations échangeables fait apparaître une saturation du complexe absorbant par $\mathrm{Al}^{3+}$ et $\mathrm{H}^{+}$, au détriment de $\mathrm{Ca}^{2+}$ et $\mathrm{Mg}^{2+}$. Le compartiment humique occupe donc une place prépondérante dans l'écosystème "pessière d'altitude" et une étude approfondie sur les interactions entre ses différentes composantes biotiques en général et le plant d'épicéa en particulier s'avère nécessaire afin de comprendre les processus responsables du dysfonctionnement de la régénération naturelle.

D'un point de vue sylvicole, il semble intéressant, les années de bonne grainée, de préparer des microsites favorables à la 
germination des graines en crochetant (voire décaper) I'humus, après avoir éliminé la végétation du sous-bois. Quant à la lutte contre les prédateurs, l'emploi de répulsifs (à trouver !) sur le site préparé, devrait permettre de limiter leur impact.

\section{REMERCIEMENTS}

Les auteurs remercient, pour leur aide financière, l'Office national des forêts et l'Association des communes forestières; pour leur appui technique, les techniciens et agents du centre ONF d'Ugine.

\section{RÉFÉRENCES}

André J, Gensac P, Pellissier F, Trosset L (1986) Régénération des peuplements d'épicéa en altitude : allélopathie et mycorhization. Rev Ecol Biol Sol 24, 3, 301-310

Bergeret $P$ (1986) La régénération de l'épicéa dans les Alpes nord-occidentales. Rôle des prédateurs. Mémoire de fin d'études BTS "Productions Forestières", 34

Bonneau M, Dambrine E, Nys $C$, Ranger J (1987) L'acidification des sols. Bull Ecol 18, $3,127-136$

Drapier $J$ (1983) Les difficultés de régénération des sapinières vosgiennes : importance de l'humus et rôle de l'allélopathie. Thèse, Univ Nancy I et INRA Champenoux, 109

Genard M, Lescourret F (1984) Les petits vertébrés de la pineraie à crochets, fonction descriptive et rôle dans la régénération forestière. L'exemple du massif d'Osséja (Pyrénées orientales). Thèse, INRA Grignon, 250

Gregori $P$ (1967) Ricerche sulla germinazione del seme di Abete bianco, Picea e Larice. Ann Accad It Sci For, 201-225
Mayer H (1986) Europäische Wälder. Ein Überblick unf Fürher durch die gefährdeten Naturwälder. UTB 1231 für Wissenschaft, Stuttgart, 384

Pellissier F, Trosset L (1989a) Effect of phytotoxic solutions on the respiration of mycorrhizal ad non-mycorrhizal spruce roots (Picea abies L Karst). Ann Sci For 46, 731s-733s

Pellissier F, Trosset $L$ (1989b) Obstacle allélopathique à la germination de graines d'épicéa et à la croissance de trois champignons ectomycorhiziens de cette essence. Acta Biol Mont IX, 153-160

Piussi $P$ (1986) La rinnovazionne della pecceta subalpina. Scienze 215, 58-67

Rost-Siebert K (1985) Untersuchungen zur $\mathrm{H}$ und Al-ionen Toxizität an Keimpflanzen von Fichte (Picea abies L Karst) und Buche (Fagus sylvatica $\mathrm{L}$ ) in Lösungskultur. Berichte des Forschungszentrums Waldökosysteme-Waldsterben, Univ Göttingen, 219

Roussel $L$ (1969) Le très jeune épicéa et la lumière. Rev For Fr 21, 1, 27-32

Smith CC, Balda RP (1979) Competition among insects, birds and mammals for conifer seeds. Am Zool 19, 1065-1083

Sorg JP (1980) Végétation et rajeunissement naturel dans la pessière subalpine de Vals (Grisons). Mémoire de I'Instit Féd de Rech Forest-EPF Zurich 56, 1, 114

Van Praag HJ, Weissen F (1985a) Aluminium effects on spruce and beech seedlings. I. Preliminary observations on plant and soil. Plant Soil 83, 331-338

Van Praag $H J$, Weissen $F$, Sougnez-Remy $S$, Carletti G (1985b) Aluminium effects on spruce and beech seedlings. II. Statistical analysis of sand culture experiments. Plant Soil 83, 339-356

Vincent JP (1977) Interactions entre les micromammifères et la production de semences forestières. Ann Sci For 34, 1, 77-87 